\title{
Treatment patterns and sequences of pharmacotherapy for patients diagnosed with depression in the United States: 2014 through 2019
}

\author{
David M. Kern ${ }^{1 *}$ (D, M. Soledad Cepeda ${ }^{1}$, Frank Defalco ${ }^{1}$ and Mila Etropolski ${ }^{2}$
}

\begin{abstract}
Background: Understanding how patients are treated in the real-world is vital to identifying potential gaps in care. We describe the current pharmacologic treatment patterns for the treatment of depression.

Methods: Patients with depression were identified from four large national claims databases during 1/1/2014-1/31/ 2019. Patients had $\geq 2$ diagnoses for depression or an inpatient hospitalization with a diagnosis of depression. Patients were required to have enrollment in the database $\geq 1$ year prior to and 3 years following their first depression diagnosis. Treatment patterns were captured at the class level and included selective serotonin reuptake inhibitors (SSRIs), serotonin and norepinephrine reuptake inhibitors, tricyclic antidepressants, other antidepressants, anxiolytics, hypnotics/sedatives, and antipsychotics. Treatment patterns were captured during all available follow-up.

Results: We identified 269,668 patients diagnosed with depression. The proportion not receiving any pharmacological treatment during follow-up ranged from 29 to $52 \%$. Of the treated, approximately half received $\geq 2$ different classes of therapy, a quarter received $\geq 3$ classes and more than 10\% received 4 or more. SSRIs were the most common first-line treatment; however, many patients received an anxiolytic, hypnotic/sedative, or antipsychotic prior to any antidepressive treatment. Treatment with a combination of classes ranged from approximately $20 \%$ of first-line therapies to $40 \%$ of fourth-line.

Conclusions: Many patients diagnosed with depression go untreated and many others receive a non-antidepressant medication class as their first treatment. More than half of patients received more than one type of treatment class during the study follow up, suggesting that the first treatment received may not be optimal for most patients.
\end{abstract}

Keywords: Depression, Treatment patterns, Antidepressants, Real-world evidence

\section{Background}

Major depressive disorder is highly prevalent in the United States, affecting more than $7 \%$ of adults and $13 \%$ of adolescents [1]. Treatment options for depression include psychotherapy, pharmacotherapy, transcranial magnetic stimulation (TMS) and invasive treatments such as electroconvulsive therapy (ECT) [2]. Treatment algorithms exist which aim to provide guidance to physicians in making effective treatment

\footnotetext{
* Correspondence: dkern2@its.jnj.com

${ }^{1}$ Janssen Research \& Development, Epidemiology, Titusville, NJ 08560, USA Full list of author information is available at the end of the article
}

decisions to improve the chances of achieving a response [3, 4]; however, these algorithms do not provide granularity about specific medications to prescribe as there are many different treatment choices that fit within the algorithm structure, though starting with a selective serotonin reuptake inhibitor (SSRI) is the most commonly recommended approach. For patients treated with pharmacotherapy, only one quarter achieve full response and remission, while a similar proportion achieve no response, and the remainder achieve partial response or response without remission [2].

Currently, there is a lack of detail about how patients diagnosed with depression are treated in the real-world. Understanding treatment patterns in a real-world setting, as 
opposed to trials in which the patterns are predetermined [5], is an important step to improving and understanding gaps in care. For example, atypical antipsychotics are the only approved adjunctive therapy for the treatment of depression [6,7], but it is unknown how often these are used or how often other off-label adjunctive treatments are used.

This paper aims to fill the gaps in knowledge about realworld pharmacologic treatment patterns of patients diagnosed with depression and the role adjunctive therapy plays.

\section{Methods}

\section{Depression cohort}

We identified patients diagnosed with depression from large national insurance claims databases during 1/1/2014 through $1 / 31 / 2019$. We chose the most recent 5 years of data, rather than a larger time period using all available data, in order to capture a snapshot of current treatment practices rather than historical treatment practices which may no longer be prevalent. More detail about the databases is found in the 'Data Source' section below. Depression was defined according to the algorithm validated by Solberg et al. [8] which required the presence of two outpatient diagnoses of depression within 365 days of each other or one inpatient depression diagnosis according to the ICD-9-CM codes $\left(269.2^{*}, 269.3^{*}, 300.4,311\right)$ or the corresponding ICD-10-CM codes (F32.", F33.", F34.1, F53.0). No exclusion for comorbid conditions, including other psychiatric conditions, was applied. The diagnosis codes used to identify depression patients do not include any codes for bipolar disorder; however, individuals with a diagnosis of bipolar disorder were not excluded from the study if they satisfied the definition of depression.

The index date was the first observed medical claim in the database with a diagnosis of depression, resulting in a cohort of newly diagnosed depression patients. Patients were required to have continuous enrollment in the database at least 1 year prior to and 3 years following the index date. Patients were excluded if they had evidence of treatment for depression - with an antidepressant or another treatment class of interest as defined below - more than 30 days prior to index, as our goal was to include patients who were newly diagnosed with depression and identify their treatment patterns from the beginning.

\section{Treatments and sequencing}

Treatment patterns were captured at the class level and included SSRIs, serotonin and norepinephrine reuptake inhibitors (SNRIs), tricyclic antidepressants (TCAs), monoamine oxidase inhibitors (MAOIs), other antidepressants (including bupropion and trazodone, among others), anxiolytics, hypnotics/sedatives, antipsychotics, psychostimulants and lithium. The individual drugs included in each class and their corresponding RxNorm ingredient codes used to identify them from the databases are found in the Appendix. Treatment sequences were captured during 30 days prior to the depression index date through all available follow-up, a minimum of 3 years. The term "treatment line" is used to describe the sequence of medication classes and combinations of medication classes received by patients during this time. Use of a specific medication class was captured at the first instance and not counted again in later lines of therapy - for example an individual filling an SSRI, switching to an SNRI, and then moving back to an SSRI would only be captured as switching from SSRI to SNRI. Because the analysis is at the class-level, in-class switching and in-class combination therapy is not captured. Drug eras were calculated as the time from the first fill for a drug in a medication class until discontinuation of that medication, allowing for gaps of up to 30 days beyond the days supply of a prescription (Fig. 1). Combination therapy with multiple classes was defined as having at least 30 days of overlap in drug eras of more than one treatment class. A fill for a medication following discontinuation of a previous drug or with fewer than 30 days of overlap was considered a switch.

\section{Data source}

The analysis was executed in four US-based administrative claims databases. Each database contains data from adjudicated health insurance claims (e.g., inpatient, outpatient/ emergency department, and outpatient pharmacy) and health plan enrollment information. Briefly, the four databases included in this study were:

1. IBM MarketScan ${ }^{\circ}$ Commercial Database (CCAE): Includes data from 142 million individuals enrolled in employer-sponsored insurance health plans. At the time of this study data were available from January 1, 2000 through January 31, 2019.

2. IBM MarketScan ${ }^{\circ}$ Multi-State Medicaid Database (MDCD): A claims database for 26 million Medicaid enrollees from multiple states. At the time of this study data were available from January 1, 2006 through June 30, 2018.

3. IBM MarketScan ${ }^{\circ}$ Medicare Supplemental Database (MDCR): Includes data for more than 9 million retirees with primary or Medicare supplemental coverage through privately insured fee-for-service, point-of-service, or capitated health plans. At the time of this study data were available from January 1, 2000 through January 31, 2019.

4. Optumఠ De-Identified Clinformatics ${ }^{\oplus}$ Data Mart Database. Includes 84 million members with private health insurance, who are fully insured in commercial plans or in administrative services only and Medicare Advantage (Medicare Advantage Prescription Drug coverage. The population is representative of US 


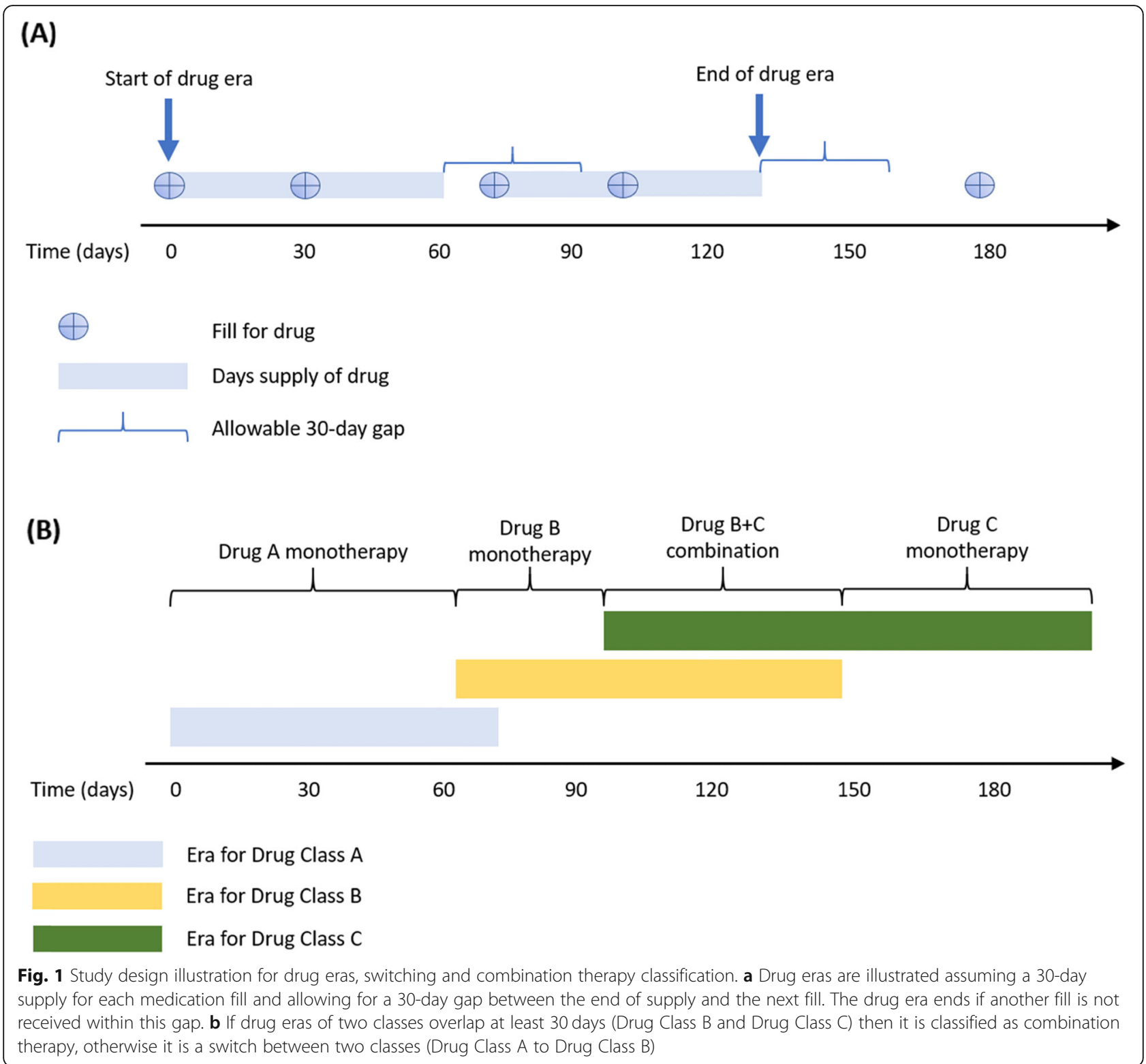

commercial claims patients ( $0-65$ years old) with some Medicare (65+ years old). At the time of this study data were available from May 31, 2000 through December 31, 2018.

Data elements included were outpatient pharmacy dispensing claims (coded with National Drug Codes), inpatient and outpatient medical claims which provide diagnosis codes (coded in ICD-9-CM or ICD-10-CM) associated with a visit. The use of the IBM MarketScan and Optum claims databases was reviewed by the New England Institutional Review Board (IRB) and was determined to be exempt from broad IRB approval, as this research project did not involve human subjects research.

\section{Baseline characteristics}

The mean age of patients (and standard deviation) on the index date within each data source, and the proportion of female patients were calculated. Comorbid conditions and the Charlson Comorbid Index [9] were captured during the year preceding, which included the index date. One diagnosis code for the comorbidity of interest was required during this time frame. Comorbidities were defined according to the Systematized Nomenclature of Medicine - Clinical Terms (SNOMED CT) classification system. The SNOMED CT classification allows mapping of various diagnostic languages across more than 80 countries, including, for example, ICD-9-CM, ICD-10-CM, and Read codes, to a single standardized set of concepts, and is used by the common data model leveraged for this study, described in the next 
section [10, 11]. Prevalence of all conditions defined by SNOMED were calculated, but only those that were most common across multiple databases and/or of special interest are reported. No statistical testing was performed to test for differences between data sources because with such a large number of patients even very small differences will be statistically significant at $p<0.05$, thus the more important inference is the qualitative difference between groups.

\section{Common data model}

Data from all four databases were mapped to standard concepts according to the Observational Medical Outcomes Partnership (OMOP) Common Data Model v5.0 [12] and the treatment sequence analysis was performed within the Observational Health Data Sciences and Informatics (or OHDSI, pronounced "Odyssey") framework. Use of the common data model allowed for consistent and precise replication across each of the four databases.

\section{Results}

A total of 269,668 patients were included from across the four databases (Table 1). The databases represent a wide range of ages, with the youngest patients from the CCAE and MDCD, while older individuals are more heavily represented in the MDCR and Optum databases. Females accounted for $62 \%$ of the patient population. Comorbid conditions such as pain, inflammatory disorders, hyperlipidemia, hypertension and diabetes were common in this population, particularly in the older Medicare population. A prior history of drug dependence was more common in the Medicaid patients (15.6\%) compared with the other databases (6.5-10.2\%), and prior evidence of suicidal thoughts was found in more than 1 in 25 Medicaid patients versus the lowest prevalence of 1 in 100 individuals from Medicare. A diagnosis for other conditions associated with the treatment classes analyzed - including bipolar disorder, post-traumatic stress disorder, psychotic disorder, obsessive compulsive disorder, and personality disorder - were relatively uncommon with most occurring in fewer than $2 \%$ of patients. One notable exception to this is the proportion of patients with a previous diagnosis of psychotic disorder in the MDCD population (6.9\%).

Roughly one-third of patients from the CCAE (29.5\%), MDCR (33.5\%), and Optum (35.9\%) databases did not receive any antidepressant or related medication during the entire follow-up period, while more than half of patients in the MDCD data (51.9\%) were untreated with pharmacotherapy (Table 2). Of patients who did receive a treatment, approximately half went on to receive a second treatment class (range across databases: 47.8-59.5\%), more than a quarter received a third (25.0-31.6\%), and more than one in ten received a fourth (10.3-15.7\%).

The most common medication class used during firstline therapy was SSRI, however there was variability in their use - more than half of patients from the CCAE database received SSRI as monotherapy or part of a combination first line treatment $(57.5 \%)$ compared with one-third of patients in the MDCD database (36.3\%) (Table 3). Non-antidepressant use made up a significant share of first-line treatments, with anxiolytics as the next most common first line treatment class received in all databases except for MDCD where hypnotic/sedative use was found in $22.6 \%$ of patients. Use of antipsychotics was not an uncommon first line approach, nearly $12 \%$ of Medicaid patients and more than 5\% of all other patients received an antipsychotic as their initial treatment. The higher prevalence of antipsychotic use in the MDCD population was likely due to a higher prevalence of comorbid psychotic disorders in this group.

Combination therapy with at least two distinct medication classes was used as the first line of treatment for 15.4$20.4 \%$ of patients, and the prevalence of combination increased in later lines of therapy, approaching $40 \%$ by the fourth line (Table 4). Use of combination therapy was relatively similar across databases, though the commercially insured populations of Optum and CCAE had slightly higher use than the MDCD and MDCR populations.

Within patients receiving monotherapy SSRI as their initial therapy, more than half of patients went on to receive a second treatment class (Table 5). Within these patients, combination of an SSRI plus another treatment class during second line occurred $27.2-31.9 \%$ of time time; however, use in combination with an antipsychotic - the only approved treatment class for adjunctive depression treatment - was found in a minority of patients (3.8-6.8\%).

The sequence of treatments within each database are shown in Fig. 2. This figure illustrates that while SSRI use was the most common first line treatment, the use of non-antidepressants - particularly anxiolytics, hypnotics/sedatives, and anticonvulsants - were common. Approximately half of patients starting on an SSRI never filled another class, while the other half moved on to a variety of different therapies. The second line therapies following first-line SSRI were not dominated by any single specific treatment class and include a mix of monotherapy treatments from other classes and the addition of a new treatments to an SSRI. The proportion of treatments accounted for by SSRIs decreased at each successive treatment line, while anticonvulsant, antipsychotic, and SNRI use steadily increases (Fig. 3); a pattern that was relatively consistent in each of the databases.

\section{Discussion}

There has been limited prior research examining depression treatment sequences and the current work substantially expands upon this groundwork. Gauthier et al. [13] examined patterns of switches, combinations, dose escalation, and discontinuation of antidepressants in general, 
Table 1 Characteristics of patients from each of the four study databases

\begin{tabular}{|c|c|c|c|c|}
\hline Characteristic & $\begin{array}{l}\text { CCAE } \\
(N=114,543)\end{array}$ & $\begin{array}{l}\text { MDCD } \\
(N=69,006)\end{array}$ & $\begin{array}{l}\text { MDCR } \\
(N=5660)\end{array}$ & $\begin{array}{l}\text { Optum } \\
(N=80,459)\end{array}$ \\
\hline Age at index (years), Mean (SD) & $32.4(15.6)$ & $37.9(25.7)$ & $74.5(8.5)$ & $51.0(23.5)$ \\
\hline Gender: Female, \% & $60.4 \%$ & $65.6 \%$ & $57.7 \%$ & $62.4 \%$ \\
\hline Charlson comorbidity index score ${ }^{a}$, Mean (SD) & $0.5(1.3)$ & $1.9(2.9)$ & $2.9(2.6)$ & $1.7(2.5)$ \\
\hline \multicolumn{5}{|l|}{ Common comorbid conditions ${ }^{a}$} \\
\hline Pain & $46.6 \%$ & $58.7 \%$ & $68.8 \%$ & $59.1 \%$ \\
\hline Inflammatory disorder of the respiratory system & $33.8 \%$ & $38.2 \%$ & $31.4 \%$ & $33.4 \%$ \\
\hline Anxiety disorder & $25.7 \%$ & $21.3 \%$ & $19.8 \%$ & $27.3 \%$ \\
\hline Traumatic injury & $21.6 \%$ & $27.4 \%$ & $30.8 \%$ & $25.4 \%$ \\
\hline Arthropathy & $20.3 \%$ & $29.8 \%$ & $51.9 \%$ & $35.9 \%$ \\
\hline Dysthymia & $20.3 \%$ & $8.6 \%$ & $17.4 \%$ & $15.7 \%$ \\
\hline Inflammatory disorder of digestive system & $20.2 \%$ & $24.1 \%$ & $18.0 \%$ & $19.1 \%$ \\
\hline Neoplastic disease & $14.8 \%$ & $10.2 \%$ & $43.6 \%$ & $24.3 \%$ \\
\hline Hyperlipidemia & $13.2 \%$ & $23.7 \%$ & $58.0 \%$ & $38.0 \%$ \\
\hline Visual system disorder & $12.7 \%$ & $31.3 \%$ & $52.8 \%$ & $31.7 \%$ \\
\hline Hypertensive disorder & $12.7 \%$ & $36.6 \%$ & $67.6 \%$ & $39.2 \%$ \\
\hline Malaise and fatigue & $12.3 \%$ & $11.9 \%$ & $22.5 \%$ & $19.2 \%$ \\
\hline Backache & $10.8 \%$ & $16.9 \%$ & $20.1 \%$ & $17.8 \%$ \\
\hline Vascular disorder & $8.1 \%$ & $19.9 \%$ & $45.9 \%$ & $24.8 \%$ \\
\hline Drug dependence & $6.5 \%$ & $15.6 \%$ & $8.0 \%$ & $10.2 \%$ \\
\hline Osteoarthritis & $5.5 \%$ & $16.5 \%$ & $34.1 \%$ & $19.6 \%$ \\
\hline Diabetes mellitus & $5.0 \%$ & $18.7 \%$ & $26.7 \%$ & $15.7 \%$ \\
\hline \multicolumn{5}{|l|}{ Other conditions of interest } \\
\hline Bipolar disorder & $1.1 \%$ & $4.3 \%$ & $1.1 \%$ & $1.5 \%$ \\
\hline PTSD & $1.8 \%$ & $3.2 \%$ & $2.2 \%$ & $1.7 \%$ \\
\hline Psychotic disorder & $1.3 \%$ & $6.9 \%$ & $3.8 \%$ & $2.7 \%$ \\
\hline Obsessive compulsive disorder & $0.8 \%$ & $0.5 \%$ & $0.5 \%$ & $0.7 \%$ \\
\hline Personality disorder & $0.8 \%$ & $1.7 \%$ & $1.1 \%$ & $0.9 \%$ \\
\hline \multicolumn{5}{|l|}{ Suicidal history } \\
\hline Suicidal thoughts & $3.9 \%$ & $4.3 \%$ & $1.0 \%$ & $2.9 \%$ \\
\hline Suicidal deliberate poisoning & $0.2 \%$ & $0.2 \%$ & $0.0 \%$ & $0.2 \%$ \\
\hline
\end{tabular}

Abbreviations: CCAE IBM MarketScan ${ }^{\oplus}$ Commercial Database, MDCD IBM MarketScan ${ }^{\oplus}$ Multi-State Medicaid Database, MDCR IBM MarketScan ${ }^{\oplus}$ Medicare Supplemental Database, SD standard deviation

${ }^{a}$ The Charlson Comorbidity Index and individual comorbidities were captured during the 365 days preceding and including the index date

Table 2 Proportion of patients who were untreated or received, at least one, two, three, or four distinct treatment lines during the entire follow-up period

\begin{tabular}{|c|c|c|c|c|c|c|c|c|}
\hline \multirow[b]{2}{*}{ Treatment line } & \multicolumn{2}{|l|}{$\begin{array}{l}\text { CCAE } \\
(N=114,543)\end{array}$} & \multicolumn{2}{|l|}{$\begin{array}{l}\text { MDCD } \\
(N=69,006)\end{array}$} & \multicolumn{2}{|l|}{$\begin{array}{l}\text { MDCR } \\
(N=5660)\end{array}$} & \multicolumn{2}{|l|}{$\begin{array}{l}\text { Optum } \\
(N=80,459)\end{array}$} \\
\hline & $\%$ of all patients & $\%$ of treated & $\%$ of all patients & $\%$ of treated & $\%$ of all patients & $\%$ of treated & $\%$ of all patients & $\%$ of treated \\
\hline Untreated & $29.5 \%$ & & $51.9 \%$ & & $33.5 \%$ & & $35.9 \%$ & \\
\hline $1 s t$ & $70.5 \%$ & $100 \%$ & $48.1 \%$ & $100 \%$ & $66.5 \%$ & $100 \%$ & $64.1 \%$ & $100 \%$ \\
\hline $2 n d$ & $42.0 \%$ & $59.5 \%$ & $23.0 \%$ & $47.8 \%$ & $35.5 \%$ & $53.4 \%$ & $34.4 \%$ & $53.7 \%$ \\
\hline $3 r d$ & $22.3 \%$ & $31.6 \%$ & $12.0 \%$ & $25.0 \%$ & $16.7 \%$ & $25.1 \%$ & $17.1 \%$ & $26.7 \%$ \\
\hline 4th & $11.1 \%$ & $15.7 \%$ & $5.9 \%$ & $12.2 \%$ & $6.8 \%$ & $10.3 \%$ & $7.8 \%$ & $12.2 \%$ \\
\hline
\end{tabular}

Abbreviations: CCAE IBM MarketScan ${ }^{\circledast}$ Commercial Database, MDCD IBM MarketScan ${ }^{\circledast}$ Multi-State Medicaid Database, $M D C R$ IBM MarketScan ${ }^{\oplus}$ Medicare Supplemental Database 
Table 3 Proportion of patients treated with each medication class out of those receiving first-line therapy (includes mono- or combination therapy)

\begin{tabular}{|c|c|c|c|c|c|c|c|c|}
\hline \multirow[b]{2}{*}{ SSRI } & \multicolumn{2}{|c|}{$\begin{array}{l}\text { CCAE } \\
(N=80,810)\end{array}$} & \multicolumn{2}{|c|}{$\begin{array}{l}\text { MDCD } \\
(N=33,186)\end{array}$} & \multicolumn{2}{|c|}{$\begin{array}{l}\text { MDCR } \\
(N=3764)\end{array}$} & \multicolumn{2}{|c|}{$\begin{array}{l}\text { Optum } \\
(N=51,585)\end{array}$} \\
\hline & 46,432 & $57.5 \%$ & 12,035 & $36.3 \%$ & 1693 & $45.0 \%$ & 25,942 & $50.3 \%$ \\
\hline Anxiolytic & 14,625 & $18.1 \%$ & 6141 & $18.5 \%$ & 695 & $18.5 \%$ & 9095 & $17.6 \%$ \\
\hline Other antidepressant & 12,196 & $15.1 \%$ & 3578 & $10.8 \%$ & 518 & $13.8 \%$ & 7438 & $14.4 \%$ \\
\hline Hypnotic/Sedative & 6981 & $8.6 \%$ & 666 & $2.0 \%$ & 99 & $2.6 \%$ & 1218 & $2.4 \%$ \\
\hline Anticonvulsant & 7377 & $9.1 \%$ & 1813 & $5.5 \%$ & 514 & $13.7 \%$ & 5855 & $11.4 \%$ \\
\hline SNRI & 4531 & $5.6 \%$ & 966 & $2.9 \%$ & 249 & $6.6 \%$ & 3145 & $6.1 \%$ \\
\hline Antipsychotic & 4609 & $5.7 \%$ & 2120 & $6.4 \%$ & 275 & $7.3 \%$ & 2944 & $5.7 \%$ \\
\hline Tricyclic & 1426 & $1.8 \%$ & 842 & $2.5 \%$ & 61 & $1.6 \%$ & 926 & $1.8 \%$ \\
\hline Stimulant & 1048 & $1.3 \%$ & 426 & $1.3 \%$ & 19 & $0.5 \%$ & 1537 & $3.0 \%$ \\
\hline Lithium & 246 & $0.3 \%$ & 106 & $0.3 \%$ & 2 & $0.1 \%$ & 110 & $0.2 \%$ \\
\hline MAOI & 7 & $0.0 \%$ & 2 & $0.0 \%$ & 3 & $0.1 \%$ & 16 & $0.0 \%$ \\
\hline
\end{tabular}

Abbreviations: CCAE IBM MarketScan ${ }^{\circledR}$ Commercial Database, MDCD IBM MarketScan ${ }^{\circledast}$ Multi-State Medicaid Database, MDCR IBM MarketScan ${ }^{\oplus}$ Medicare

Supplemental Database, SSRI Selective serotonin reuptake inhibitor, SNRI Serotonin and norepinephrine reuptake inhibitor, MAOI Monoamine oxidase inhibitor

but did not look at individual drugs or classes, and did not include non-antidepressant treatment classes. Hripcsak et al. [14], used a similar methodology to identify depression treatment sequences in multiple databases; however, this analysis did not capture combination therapy or use of non-antidepressant medication classes, among other differences in the approach.

Our study leveraged prescription claims data from four patient populations representing a broad cross-section of the US population, including commercially insured individuals, those receiving Medicare, and individuals on Medicaid. There was a relatively low prevalence of first-line SSRI use (occurring in less than half of patients) in contrast to many of the treatment guidelines recommending starting with monotherapy SSRI $[15,16]$. Furthermore, use of anxiolytics, anticonvulsants, and hypnotics/sedatives were commonly used as the first treatment choice in these patients newly diagnosed with depression, potentially pointing to a reluctancy of physicians to prescribe antidepressants [17] but being more comfortable using other classes of drugs such as anxiolytics $[18,19]$. High use of benzodiazepines, which comprised the majority of anxiolytic use in this study, is concerning because they are not recommended as

Table 4 Prevalence of combination therapy during each treatment line within treated patients

\begin{tabular}{lllll}
\hline Treatment line & CCAE & MDCD & MDCR & Optum \\
\hline 1st & $20.4 \%$ & $17.9 \%$ & $15.4 \%$ & $18.3 \%$ \\
2nd & $32.7 \%$ & $32.2 \%$ & $30.7 \%$ & $33.9 \%$ \\
3rd & $36.1 \%$ & $35.1 \%$ & $32.8 \%$ & $37.9 \%$ \\
4th & $38.6 \%$ & $38.6 \%$ & $31.9 \%$ & $40.0 \%$ \\
\hline
\end{tabular}

Abbreviations: CCAE IBM MarketScan ${ }^{\circledast}$ Commercial Database, MDCD IBM MarketScan ${ }^{\oplus}$ Multi-State Medicaid Database, MDCR IBM MarketScan ${ }^{\circledast}$ Medicare Supplemental Database a first-line therapy [15] and they carry concerns of abuse [20-22] and risk of overdose [23, 24].

This study showed that while general trends across these populations were relatively similar there were some important differences. Specifically, patients covered by Medicaid tended to have treatment patterns that were different than the other three groups - more than half of patients diagnosed with depression were untreated, first-line SSRI use was lower, and use of alternative treatment classes outside of antidepressants occurred more often. The Medicaid sample represents a population of vulnerable individuals of lower socio-economic status and high burden of disease and it appears they are receiving different care when compared with the other patient populations.

The results of this real-world assessment of treatment practices appear to contradict some common treatment recommendations regarding treatment with pharmacotheapy. Many patients, ranging from one-third to one-half, received no pharmacotherapy for their depression during the entire follow-up, a period covering a minimum of 3 years in all patients. This was not limited to only Medicaid patients, as mentioned above, but also affected patients from the other databases. More so, this could be an underestimate of the true prevalence of untreated depression patients, because a significant proportion of individuals go undiagnosed and therefore are not able to receive treatment. Previous research screening individuals for depression rather than relying on a physician diagnosis has found that just $29-46 \%$ received a treatment for their depression $[25,26]$. The results found in our study may be due to patients receiving alternative forms of treatment, such as psychotherapy, rather than pharmacologic treatment. It may also be that individuals who did not have dispensings had less severe depression or were prescribed non-pharmacological interventions. The American College of Physicians recommends clinicians 
Table 5 Treatment patterns of those receiving first line monotherapy SSRI treatment

\begin{tabular}{|c|c|c|c|c|c|c|c|c|}
\hline \multirow[b]{2}{*}{ Received first line SSRI (monotherapy) } & \multicolumn{2}{|l|}{ CCAE } & \multicolumn{2}{|c|}{ MDCD } & \multicolumn{2}{|c|}{ MDCR } & \multicolumn{2}{|c|}{ Optum } \\
\hline & 34,453 & & 8292 & & 1341 & & 19,588 & \\
\hline $\begin{array}{l}\text { Patients on first line SSRI monotherapy who went on to receive } \\
\text { second treatment class }\end{array}$ & 19,231 & $55.8 \%$ & 4812 & $58.0 \%$ & 703 & $52.4 \%$ & 10,090 & $51.5 \%$ \\
\hline Second line is combination therapy & 7814 & $40.6 \%$ & 1784 & $37.1 \%$ & 265 & $37.7 \%$ & 4034 & $40.0 \%$ \\
\hline Second line is combination therapy which includes an SSRI & 6085 & $31.6 \%$ & 1309 & $27.2 \%$ & 224 & $31.9 \%$ & 3200 & $31.7 \%$ \\
\hline Second line is combination therapy of SSRI + antipsychotic & 828 & $4.3 \%$ & 325 & $6.8 \%$ & 31 & $4.4 \%$ & 385 & $3.8 \%$ \\
\hline
\end{tabular}

Abbreviations: CCAE IBM MarketScan ${ }^{\oplus}$ Commercial Database, MDCD IBM MarketScan ${ }^{\bullet}$ Multi-State Medicaid Database, MDCR IBM MarketScan ${ }^{\oplus}$ Medicare Supplemental Database, SSRI Selective serotonin reuptake inhibitor

choose between cognitive behavior therapy or secondgeneration antidepressants after discussing the pros and cons of the treatment choices with their patient [27]. And the American Psychiatric Association (APA) recommends psychotherapy alone as an initial treatment for patients with mild to moderate major depressive disorder [15].
The high prevalence of non-antidepressant treatment classes could reflect the high rates of comorbid conditions, such as anxiety disorder or sleep disorders [28]; however, these medications are largely being prescribed as monotherapy and not in combination with an antidepressant.

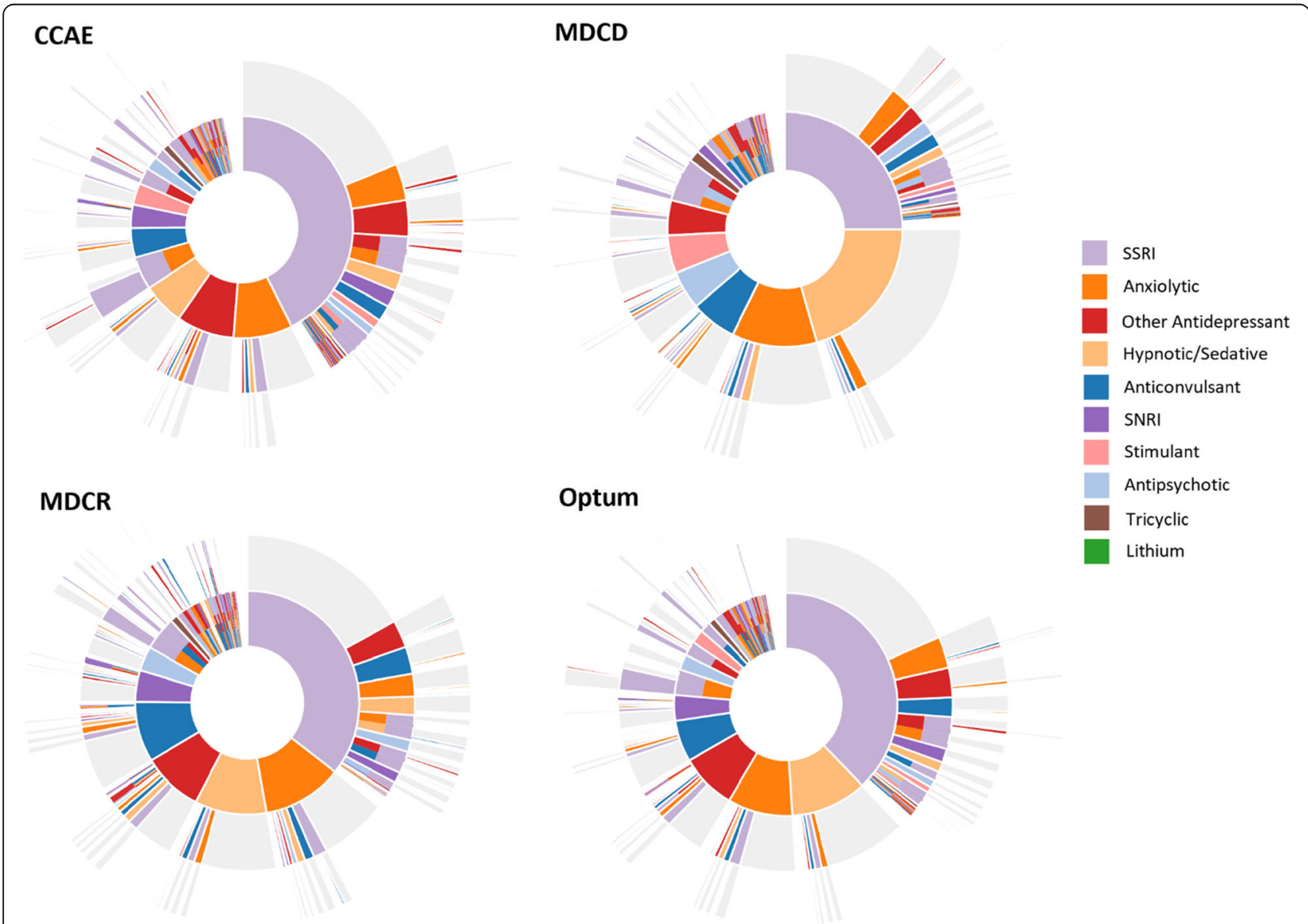

Fig. 2 Sunburst of treatment patterns starting with first line (inner-most donut) to fourth line (outer slices). Each color represents a distinct treatment class, and each layer repesents a new treatment line and illustrate the sequence in which patients received different therapies; for example the large orange piece in the middle indicates first-line SSRI use, and the pink slice on the next outter ring adjacent to the orange indicates a switch from an SSRI to an anxiolytic. Slices that have multiple colors indicate combination therapy with more than one medication class. Slices in grey indicate no additional medication was taken. Abbreviations: CCAE, IBM MarketScan ${ }^{\oplus}$ Commercial Database; MDCD, IBM MarketScan ${ }^{\oplus}$ Multi-State Medicaid Database; MDCR, IBM MarketScan ${ }^{\circledR}$ Medicare Supplemental Database; SSRI, selective serotonin reuptake inhibitor; SNRI, serotonin and norepinephrine reuptake inhibitor 


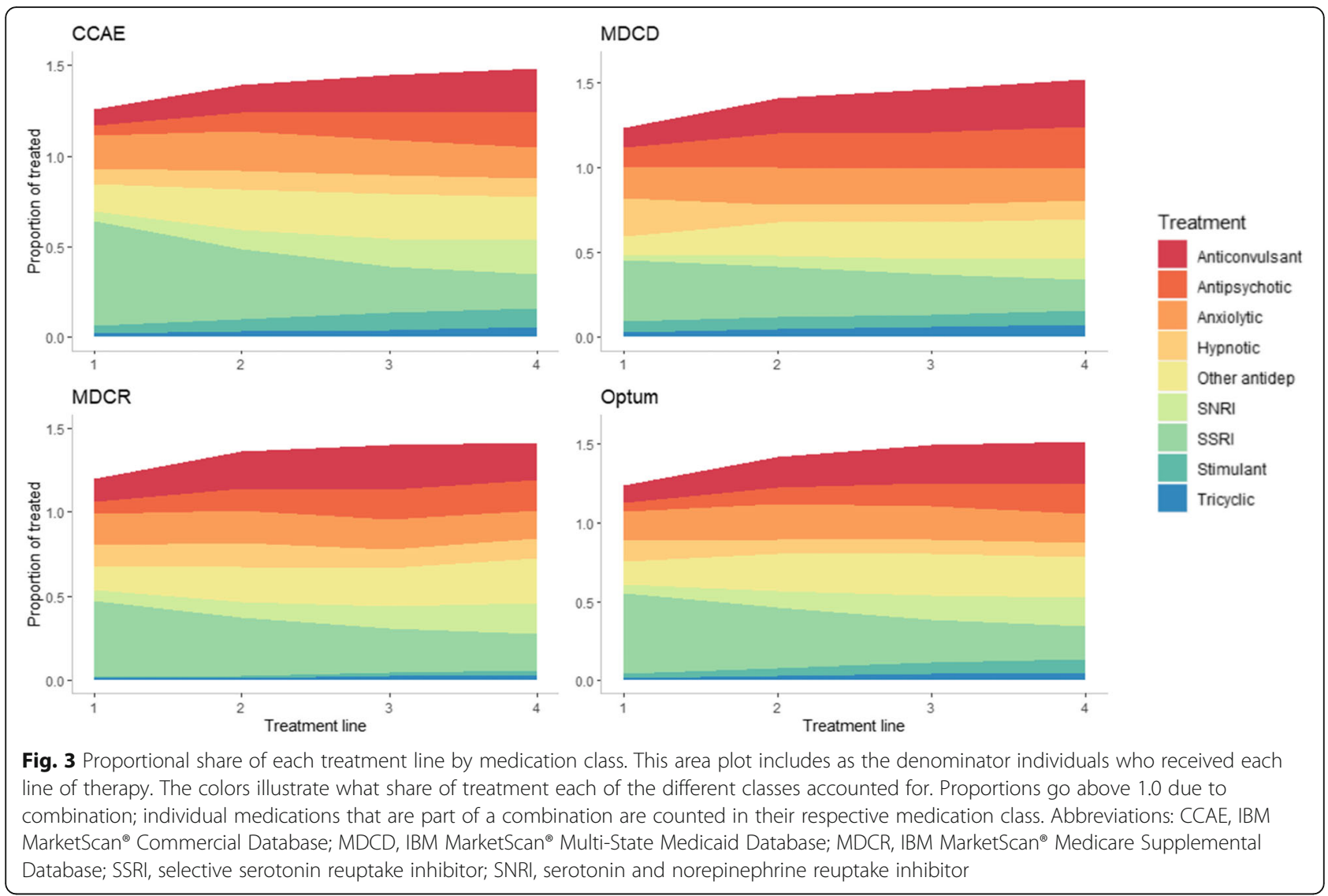

\section{Limitations}

There are limitations to this study. This analysis focused only on pharmacotherapy for the treatment of depression and did not examine rates of psychotherapy or procedures such as ECT or TMS, which play an important role in the overall care patients receive, and may account for the proportion of patients that were classified as untreated. Patients with depression were identified using diagnoses codes which are not a perfect tool; however, we used a previously published algorithm for identifying depression in claims data which achieved high validity $(\mathrm{PPV}=99 \%)$ [8]. Because the algorithm requires two outpatient or one inpatient diagnosis, there is less of a chance of falsely classifying a patient as having depression due to a rule-out or misdiagnosis that may happen if only requiring a single diagnosis. However, we do not capture depression patients who received only a single diagnosis of depression in an outpatient setting. This is a trade-off we deliberately made to improve certainty that we only included subjects with depression.

This analysis did not capture any within-class switching or combination; for example, receiving two SSRIs is simply captured as monotherapy SSRI use and switching from one SSRI to another does not appear as a change in therapy. This is because the goal of this study was to understand the order in which different therapy classes are first received over time, but this provides opportunity for future research to look in detail at the individual drug level to assess in-class treatment changes.

This study did not examine the average time patients were actively receiving each treatment, or how long patients may have been with no treatment between switching from one class to the next, as it was outside of the scope of this research. Discontinuation of antidepressant treatment is common and has been identified as a risk factor for relapses [29-33].

There is no diagnosis associated with prescription claims, thus receiving treatment for non-antidepressant classes is not guaranteed to have been prescribed for treating the underlying depression or its symptoms. To mitigate this misclassification due to receiving therapy for reasons unrelated to depression, we required treatment to occur at the time of or following the first diagnosis of depression with no prior history of treatment in the database; however, this does not guarantee that treatments could not have been for other conditions that began treatment following a patient's first depression diagnosis. Additionally, the pharmacy claims are a record of medication dispensed to a patient, they do not capture prescriptions that were written by a physician but never filled by the patient. 
Our study required three-years of continuous observation following the index depression diagnosis. This followup requirement was chosen to capture sufficient followup across the population to allow us to see multiple lines of therapy and various treatment changes. It's possible, and even likely, that by doing so we are excluding a certain subset of individuals with depression, but the alternative of requiring too short of a follow-up period would have prevented us from seeing what happens during later lines of therapy and would distort the observed treatment patterns in the population as a whole. By making this decision we sacrificed broader generalizability of results but increased the validity of what was observed.

\section{Strengths}

This study included more than a quarter-million individuals diagnosed with depression across four major claims databases representing a full-spectrum of ages and types of insurance coverage. When examined together, these databases provide generalizability to a broad cross-section of the United States. We were able to leverage the infrastructure of the common data model and the tools from the OHDSI network to achieve a uniform and consistent approach across each of these four databases whose underlying data structures differ. This work expands upon previous work by not limiting the analysis to only drugs that are specifically classified as antidepressants. It is widely known that medications in various other classes are commonly used to treat patients with depression and this study reflects real-world prescribing practices.

\section{Conclusions}

This study provides the most detailed reflection of realworld pharmacotherapy practices for the treatment of depression in the United States to date. Understanding how depression patients are being managed is an important first step in improving care.

\section{Abbreviations \\ APA: American Psychiatric Association; CCAE: IBM MarketScan ${ }^{\oplus}$ Commercial Database; ECT: Electroconvulsive therapy; ICD-10-CM: International Classification of Diseases, Tenth Revision, Clinical Modification; ICD-9- CM: International Classification of Diseases, Ninth Revision, Clinical Modification; IRB: Institutional Review Board; MAOI: Monoamine oxidase inhibitor; MDCD: IBM MarketScan ${ }^{\oplus}$ Multi-State Medicaid Database; MDCR: IBM MarketScan ${ }^{\circledast}$ Medicare Supplemental Database; OHDSI: Observational Health Data Sciences and Informatics; OMOP: Observational Medical Outcomes Partnership; SNRI: Serotonin and norepinephrine reuptake inhibitor; SSRI: Selective serotonin reuptake inhibitor; TCA: Tricyclic antidepressant; TMS: Transcranial magnetic stimulation}

\section{Acknowledgements}

Not applicable.

\section{Authors' contributions}

DMK contributed to the study design, data acquisition and analysis, interpretation of results, and writing and revising the manuscript. MSC contributed to the study design, interpretation of results, and reviewing the manuscript. FD contributed to data acquisition, data analysis, and reviewing of the manuscript. ME contributed to the study design, interpretation of results, and reviewing the manuscript. All authors read and approved the final manuscript.

\section{Funding}

Janssen Research \& Development, LLC funded this research and led all aspects of the study, including the design, analysis, interpretation of data, and writing of the manuscript.

Availability of data and materials

Data are available on request.

\section{Ethics approval and consent to participate}

The use of the databases was reviewed by the New England Institutional Review Board (IRB) and was determined to be exempt from broad IRB approval, as this research project did not involve human subjects research.

\section{Consent for publication}

Not applicable.

\section{Competing interests}

All authors are employees of Janssen Research \& Development, LLC, which markets and develops treatments for depression, and are stockholders of Johnson \& Johnson, Janssen's parent company.

\section{Author details}

'Janssen Research \& Development, Epidemiology, Titusville, NJ 08560, USA. 2Janssen Research \& Development, Neuroscience TA, Titusville, NJ 08560, USA.

Received: 1 October 2019 Accepted: 22 December 2019 Published online: 03 January 2020

\section{References}

1. National Institute of Mental Health. Major Depression 2019 [Last Accessed: September 9, 2019]. Available from: https://www.nimh.nih.gov/health/ statistics/major-depression.shtml.

2. Fava M, Rush AJ, Trivedi MH, Nierenberg AA, Thase ME, Sackeim HA, et al. Background and rationale for the sequenced treatment alternatives to relieve depression (STAR*D) study. Psychiatr Clin North Am. 2003;26(2):457-94 x.

3. Adli M, Wiethoff K, Baghai TC, Fisher R, Seemuller F, Laakmann G, et al. How effective is algorithm-guided treatment for depressed inpatients? Results from the randomized controlled multicenter German algorithm project 3 trial. Int J Neuropsychopharmacol. 2017;20(9):721-30.

4. Trivedi MH, Kleiber BA. Algorithm for the treatment of chronic depression. J Clin Psychiatry. 2001;62(Suppl 6):22-9.

5. Gaynes BN, Rush AJ, Trivedi MH, Wisniewski SR, Spencer D, Fava M. The STAR*D study: treating depression in the real world. Cleve Clin J Med. 2008; 75(1):57-66

6. Wang P, Si T. Use of antipsychotics in the treatment of depressive disorders. Shanghai Arch Psychiatry. 2013;25(3):134-40

7. Wang S-M, Han C, Lee S-J, Jun T-Y, Patkar AA, Masand PS, et al. Second generation antipsychotics in the treatment of major depressive disorder: an update. Chonnam Med J. 2016;52(3):159-72

8. Solberg LI, Engebretson Kl, Sperl-Hillen JM, Hroscikoski MC, O'Connor PJ. Are claims data accurate enough to identify patients for performance measures or quality improvement? The case of diabetes, heart disease, and depression. Am J Med Qual. 2006;21(4):238-45.

9. Romano PS, Roos LL, Jollis JG. Adapting a clinical comorbidity index for use with ICD-9-CM administrative data: differing perspectives. J Clin Epidemiol. 1993;46(10):1075-90.

10. Hripcsak G, Levine ME, Shang N, Ryan PB. Effect of vocabulary mapping for conditions on phenotype cohorts. J Am Med Inform Assoc. 2018:25(12): $1618-25$.

11. SNOMED CT. SNOMED CT 5-Step Briefing 2019. Available from: http://www. snomed.org/snomed-ct/five-step-briefing.

12. Stang PE, Ryan PB, Racoosin JA, Overhage JM, Hartzema AG, Reich C, et al. Advancing the science for active surveillance: rationale and design for the observational medical outcomes partnership. Ann Intern Med. 2010;153(9):600-6.

13. Gauthier G, Guérin A, Zhdanava M, Jacobson W, Nomikos G, Merikle E, et al. Treatment patterns, healthcare resource utilization, and costs following first- 
line antidepressant treatment in major depressive disorder: a retrospective US claims database analysis. BMC Psychiatry. 2017;17(1):222.

14. Hripcsak G, Ryan PB, Duke JD, Shah NH, Park RW, Huser V, et al. Characterizing treatment pathways at scale using the OHDSI network. Proc Natl Acad Sci U S A. 2016;113(27):7329-36.

15. American Psychiatric Association. Practice Guidelines for the Treatment of Patients with Major Depressive Disorder 2010 [Last Accessed: August 19, 2019]. Available from: https://psychiatryonline.org/pb/assets/raw/sitewide/ practice_guidelines/guidelines/mdd.pdf.

16. Gelenberg AJ. A review of the current guidelines for depression treatment. J Clin Psychiatry. 2010;71(7):e15.

17. Hyde J, Calnan M, Prior L, Lewis G, Kessler D, Sharp D. A qualitative study exploring how GPs decide to prescribe antidepressants. Br J Gen Pract. 2005;55(519):755-62.

18. Haw C, Stubbs J. Benzodiazepines--a necessary evil? A survey of prescribing at a specialist UK psychiatric hospital. J Psychopharmacol. 2007;21 (6):645-9.

19. Sirdifield C, Anthierens S, Creupelandt H, Chipchase SY, Christiaens T, Siriwardena AN. General practitioners' experiences and perceptions of benzodiazepine prescribing: systematic review and meta-synthesis. BMC Fam Pract. 2013;14(1):191.

20. Brett J, Murnion B. Management of benzodiazepine misuse and dependence. Aust Prescr. 2015;38(5):152-5.

21. Lader M. Benzodiazepines revisited--will we ever learn? Addiction. 2011; 106(12):2086-109.

22. Votaw VR, Geyer R, Rieselbach MM, McHugh RK. The epidemiology of benzodiazepine misuse: a systematic review. Drug Alcohol Depend. 2019; 200:95-114.

23. Park TW, Saitz R, Ganoczy D, llgen MA, Bohnert AS. Benzodiazepine prescribing patterns and drug overdose mortality among individuals receiving opioid analgesics. Addict Sci Clin Pract. 2015;10(1):A48.

24. Sun EC, Dixit A, Humphreys K, Darnall BD, Baker LC, Mackey S. Association between concurrent use of prescription opioids and benzodiazepines and overdose: retrospective analysis. BMJ. 2017;356:j760.

25. Olfson M, Blanco C, Marcus SC. Treatment of adult depression in the United States. JAMA Intern Med. 2016;176(10):1482-91.

26. Cepeda MS, Stang P, Makadia R. Depression is associated with high levels of C-reactive protein and low levels of fractional exhaled nitric oxide: results from the 2007-2012 National Health and nutrition examination surveys. J Clin Psychiatry. 2016;77(12):1666-71.

27. Qaseem A, Barry MJ, Kansagara D. Nonpharmacologic versus pharmacologic treatment of adult patients with major depressive disorder: a clinical practice guideline from the American College of Physicians. Ann Intern Med. 2016;164(5):350-9.

28. Cepeda MS, Stang P, Blacketer C, Kent JM, Wittenberg GM. Clinical relevance of sleep duration: results from a cross-sectional analysis using NHANES. $J$ Clin Sleep Med. 2016;12(6):813-9.

29. Keyloun KR, Hansen RN, Hepp Z, Gillard P, Thase ME, Devine EB. Adherence and persistence across antidepressant therapeutic classes: a retrospective claims analysis among insured US patients with major depressive disorder (MDD). CNS drugs. 2017;31(5):421-32.

30. Olfson M, Marcus SC, Tedeschi M, Wan GJ. Continuity of antidepressant treatment for adults with depression in the United States. Am J Psychiatry. 2006;163(1):101-8

31. Fagot JP, Cuerq A, Samson S, Fagot-Campagna A. Cohort of one million patients initiating antidepressant treatment in France: 12-month follow-up. Int J Clin Pract. 2016;70(9):744-51.

32. Kim MJ, Kim N, Shin D, Rhee SJ, Park CHK, Kim H, et al. The epidemiology of antidepressant use in South Korea: does short-term antidepressant use affect the relapse and recurrence of depressive episodes? PLoS One. 2019; 14(9):e0222791.

33. Mars B, Heron J, Kessler D, Davies NM, Martin RM, Thomas KH, et al. Influences on antidepressant prescribing trends in the UK: 1995-2011. Soc Psychiatry Psychiatr Epidemiol. 2017;52(2):193-200.

\section{Publisher's Note}

Springer Nature remains neutral with regard to jurisdictional claims in published maps and institutional affiliations.

Ready to submit your research? Choose BMC and benefit from:

- fast, convenient online submission

- thorough peer review by experienced researchers in your field

- rapid publication on acceptance

- support for research data, including large and complex data types

- gold Open Access which fosters wider collaboration and increased citations

- maximum visibility for your research: over $100 \mathrm{M}$ website views per year

At BMC, research is always in progress.

Learn more biomedcentral.com/submissions 